\title{
A novel brainstem tumor model: guide screw technology with functional, radiological, and histopathological characterization
}

\author{
James Lee, B.S., George I. Jallo, M.D., Michael Guarnieri, Ph.D., \\ Benjamin S. Carson Sr., M.D., and Margret B. Penno, Ph.D. \\ Department of Neurosurgery, Johns Hopkins University School of Medicine, Baltimore, Maryland
}

\begin{abstract}
Object. Survival rates for high-grade brainstem tumors are approximately $10 \%$ and optimal therapy has yet to be determined. Development of a satisfactory brainstem tumor model is necessary for testing new therapeutic paradigms that may prolong survival. The authors report the technique, functional progression, radiological appearance, and histopathological features of a novel brainstem tumor model in rats.

Methods. Thirty female Fischer 344 rats were randomized (10 animals/group) to receive an injection of either $3 \mu l$ of 9L gliosarcoma cells (100,000 cells), $3 \mu \mathrm{l}$ of F98 glioma cells (100,000 cells), or $3 \mu \mathrm{l}$ of medium (Dulbecco modified Eagle medium) into the pontine tegmentum of the brainstem. Using a cannulated guide screw system implanted in the skull of the animal, rats in each group were injected at coordinates $1.4 \mathrm{~mm}$ to the right of the sagittal and $1 \mathrm{~mm}$ anterior to the lambdoid sutures, at a depth of $7 \mathrm{~mm}$ from the dura mater. The angle of the syringe during injection was anteflexed $5^{\circ}$ from the vertical. Postoperatively, the rats were evaluated for neurological deficits by using an automated rotarod test. High-resolution $\left[{ }^{18} \mathrm{~F}\right]$ fluorodeoxyglucose-positron emission tomography (FDG-PET) fused with computerized tomography (CT) scans were acquired pre- and postoperatively through the onset of hemiparesis and correlated accordingly. Kaplan-Meier curves were generated for survival and disease progression, and brains were processed postmortem for histopathological investigation.

The 9L and F98 tumor cells grew in 95\% of the animals in which they were injected and resulted in a statistically significant mean onset of hemiparesis of $16.5 \pm 0.56$ days $(p=0.001, \log$-rank test), compared with animals in the control group, which had no neurological deficits by Day 45. The FDG-PET studies coregistered with CT scans demonstrated space-occupying brainstem lesions, and this finding was confirmed by histological studies. Animals in the control group showed no functional, radiological, or pathological signs of tumor.

Conclusions. Progression to hemiparesis was consistent in all tumor-injected animals, with predictable onset of symptoms occurring approximately 17 days postsurgery. The histopathological and radiological characteristics of the 9L and F98 brainstem tumors were comparable to those of aggressive primary human brainstem tumors. Establishment of this animal tumor model will facilitate the testing of new therapeutic paradigms for the treatment of these lesions.
\end{abstract}

KEY WORDS • brainstem tumor • pontine glioma • animal model • rat

Brainstem tumors are rare lesions that comprise approximately 10 to $15 \%$ of all CNS tumors in children. ${ }^{3,8}$ The most common types of brainstem tumors are diffuse intrinsic gliomas. ${ }^{1,5}$ These diffuse, inoperable lesions have a poor prognosis and cannot be treated with resection. Current treatment options for these high-grade and diffuse tumors are limited to radiotherapy with or without adjuvant chemotherapy. Despite multimodal adjuvant therapy, patients with malignant brainstem tumors die of their disease within a relatively short time, usually 12 months from the time of diagnosis. ${ }^{2,12}$

Although chemotherapy has been incorporated into the treatment of various intracranial neoplasms, the role of che-

Abbreviations used in this paper: $\mathrm{CNS}=$ central nervous system; $\mathrm{CT}=$ computerized tomography; DMEM = Dulbecco modified Eagle medium; FDG-PET $=\left[{ }^{18} \mathrm{~F}\right]$ fluorodeoxyglucose-positron emission tomography. motherapeutic agents for brainstem tumors remains undefined. Systemic chemotherapy has been used in the treatment of these inoperable tumors, ${ }^{13}$ but the efficacy of this approach needs to be confirmed with larger studies. The discovery of more potent and specific antineoplastic agents, and the development of novel drug delivery systems for CNS malignancies, including controlled-release polymers, ${ }^{7,17}$ local therapy, ${ }^{4,6}$ and convection-enhanced delivery, ${ }^{14}$ among others, could benefit the treatment of brainstem tumors. Nevertheless, testing of these novel treatments requires a reproducible and accessible animal model of the tumors for preclinical investigation.

The current literature lacks a comprehensive animal model for brainstem tumors, and development of such a model is a prerequisite for future investigations. An ideal animal model for this disease should be accessible and have a reproducible course of onset of paraparesis, with a pre- 
dictable pattern of tumor infiltration and a therapeutic window adequate for experimental intervention; it must be amenable to radiological monitoring and also mimic the infiltrative nature of human brainstem tumors. In this study we describe a novel brainstem tumor model in rats in which the 9L gliosarcoma and F98 glioma cell lines are used, and we report the onset of hemiparesis, results of radiographic studies, and histopathological characteristics of the tumor model. To our knowledge, this is the first animal model of brainstem tumors with a predictable onset of hemiparesis, fused PET/CT imaging, and histopathological analysis.

\section{MATERIALS AND METHODS}

\section{Experimental Design}

Thirty female Fischer 344 rats were randomized into two experimental groups and one control group, each containing 10 animals. Animals in the tumor groups received a 3$\mu \mathrm{l}$ injection of $10^{5}$ cells $/ \mathrm{ml}(100,000$ cells $)$ of either F98 glioma or 9L gliosarcoma cells at the same concentration and coordinates. Animals in the control group received a 3$\mu l$ injection of DMEM at the same level. The rats were followed clinically, and radiological analysis as described later and the onset of hemiparesis were documented. Cognitive and functional deficits were scored using an automated rotarod test. ${ }^{16}$ The brains were processed postmortem to analyze histopathological features.

\section{Experimental Animals}

Female Fischer 344 rats (Charles River Laboratories, Wilmington, MA) weighing between 150 and $200 \mathrm{~g}$ (mean $180 \mathrm{~g}$ ) were used in this experiment. The rats were housed in standard facilities and given free access to Baltimore City water and rat chow. The experimental protocol was approved by the Animal Care and Use Committee of the Johns Hopkins University and met all federal guidelines.

\section{Tumor Cell Lines}

The 9L and F98 tumor cell lines were maintained in the Johns Hopkins University Genetics CORE Cell Center facilities. The tumor cells were prepared for injection by using the standard cell preparation protocol, centrifuged, and resuspended in DMEM. Viable cells, which were determined by trypan blue exclusion, were counted with a hemocytometer. Cells were diluted to a concentration of $10^{5}$ cells $/ 3 \mu l$.

\section{Surgical Technique}

Animals were anesthetized with an intramuscular injection of a solution of $50 \mathrm{mg} / \mathrm{kg}$ ketamine $(100 \mathrm{mg} / \mathrm{ml}$; Abbott Laboratories, Chicago, IL) and $10 \mathrm{mg} / \mathrm{kg}$ xylazine (100 mg/ml; Phoenix Pharmaceutical, St. Joseph, MO). The posterior cranial region was shaved and prepared in a sterile fashion. A midline incision approximately $2 \mathrm{~cm}$ long was cut in the skin over the skull, and a burr hole was made using steel drill bits (Plastics One, Roanoke, VA) at coordinates $1.4 \mathrm{~mm}$ right of the sagittal and $1 \mathrm{~mm}$ anterior to the lambdoid suture. The cannulated guide screws (Plastics One) were inserted into the burr holes for cell implantation. A 22-gauge 10- $\mu$ l Hamilton needle (Hamilton Co., Reno, $\mathrm{NV}$ ) was inserted at an angle $5^{\circ}$ anteflexed from horizontal to a depth of $7 \mathrm{~mm}$ from the dura mater (Fig. 1A). The 20 rats in the tumor groups received a $3-\mu l$ brainstem injection of $10^{5}$ 9L gliosarcoma or F98 glioma cells (Fig. 1B), whereas the 10 in the control group received a $3-\mu 1$ injection of DMEM (Table 1). To avoid cell leakage or backflow of solvent, a stylet plug was inserted into the guide screw and the incision was closed with surgical clips in a standard fashion. After recovery, the rats were returned to their cages. Each animal was subsequently evaluated for neurological deficits.
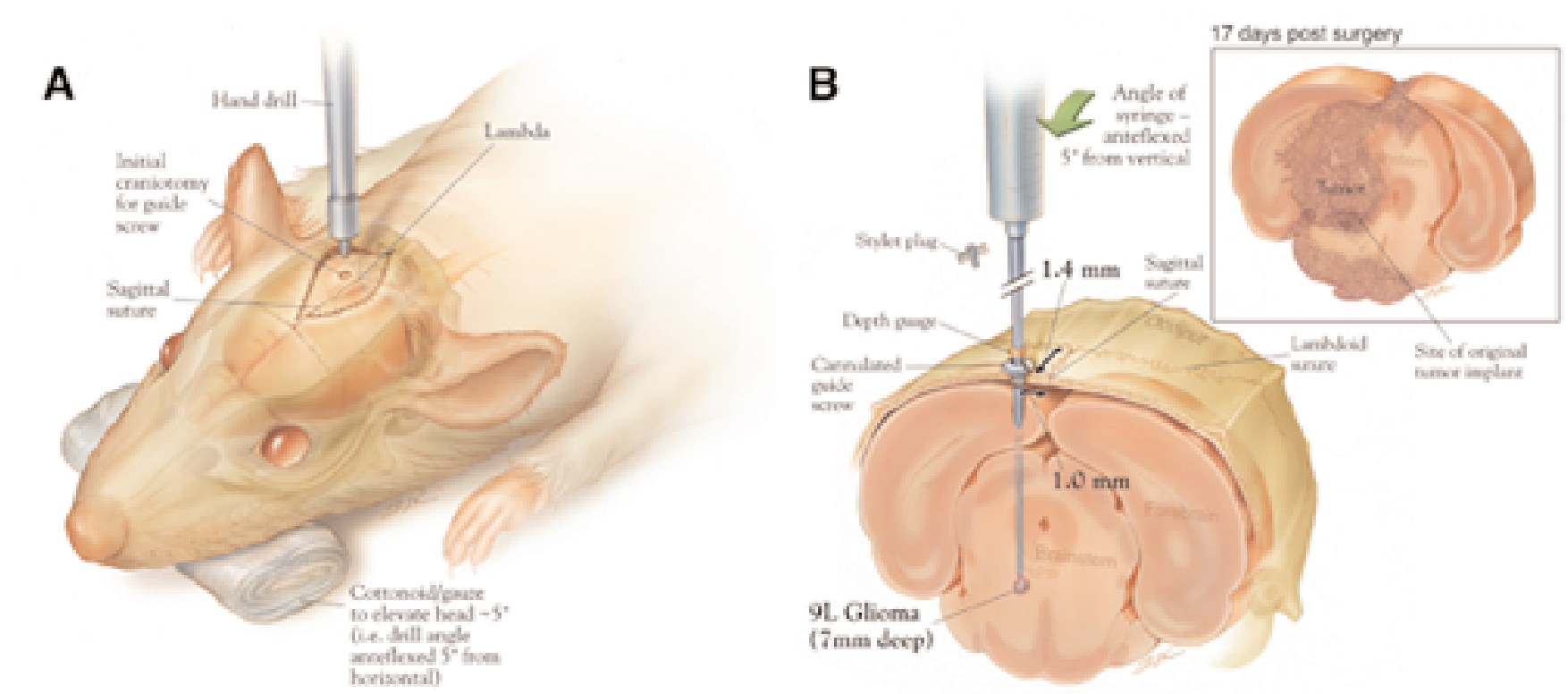

Fig. 1. A: Artist's illustration showing the position of the rat and angle for tumor implantation. B: Artist's rendering showing the anatomy of the rat brainstem, location that is resected, the trajectory of the needle, and the location of tumor implantation. 


\section{TABLE 1}

Chart showing the onset of paresis (in days) and survival for animals used in the experiment in rats injected with tumor cells or DMEM

\begin{tabular}{|c|c|c|}
\hline Animal Group & Onset of Hemiparesis & Day of Death \\
\hline \multicolumn{3}{|l|}{ control } \\
\hline 1 & 60 & 60 \\
\hline 2 & 60 & 60 \\
\hline 3 & 60 & 60 \\
\hline 4 & 60 & 60 \\
\hline 5 & 60 & 60 \\
\hline 6 & 60 & 60 \\
\hline 7 & 60 & 60 \\
\hline 8 & 60 & 60 \\
\hline 9 & 60 & 60 \\
\hline 10 & 60 & 60 \\
\hline \multicolumn{3}{|l|}{$9 L$} \\
\hline 1 & 15 & 16 \\
\hline 2 & 16 & 17 \\
\hline 3 & 15 & 17 \\
\hline 4 & 16 & 18 \\
\hline 5 & 16 & 18 \\
\hline 6 & 17 & 18 \\
\hline 7 & 17 & 19 \\
\hline 8 & 18 & 19 \\
\hline 9 & 21 & 22 \\
\hline 10 & 20 & 22 \\
\hline \multicolumn{3}{|l|}{ F98 } \\
\hline 1 & 15 & 17 \\
\hline 2 & 14 & 17 \\
\hline 3 & 15 & 17 \\
\hline 4 & 16 & 17 \\
\hline 5 & 16 & 17 \\
\hline 6 & 15 & 17 \\
\hline 7 & 16 & 18 \\
\hline 8 & 17 & 18 \\
\hline 9 & 19 & 22 \\
\hline 10 & 60 & 60 \\
\hline
\end{tabular}

\section{Imaging Analysis}

All animals underwent high-resolution FDG-PET and CT scanning preoperatively as well as during the study period. Before scans were obtained, the animals were sedated with intramuscular injections of $50 \mathrm{mg} / \mathrm{kg}$ ketamine and 10 $\mathrm{mg} / \mathrm{kg}$ xylazine, and an intravenous tail injection of $\left[{ }^{18} \mathrm{~F}\right] \mathrm{FDG}$ at a concentration of $500 \pm 15 \mu \mathrm{Ci}$ was given. Imaging beds were placed over the brain at positions $9 \mathrm{~mm}$ above and below the screw. Thirty minutes after injection of $\left[{ }^{18} \mathrm{~F}\right] \mathrm{FDG}$, the animals underwent scanning for which two imaging beds were used. The length of time in each bed was equal to 10 minutes, for a total duration of 20 minutes. The rats were kept under sedation with isoflurane gas at a rate of $1 \% \mathrm{gas} / \mathrm{L} / \mathrm{min}$. The CT scans were acquired with an X-Spect Dedicated Animal Imaging scanner and LumaGEM acquisition program (Gamma Medica, Northridge, CA), which acquired images in 256 projections by using cone-beam geometry and reconstructed them with filtered back-projection. The FDG-PET images were acquired on a small-animal PET scanner (ATLAS; National Institutes of Health, Bethesda, MD) with a $250-700 \mathrm{keV}$ energy window and a $1.8-\mathrm{cm}$ transaxial field of view. Images were reconstructed with a three-dimensional orderedsubsets expectation-maximization algorithm by using four iterations and four subsets. The FDG-PET images were then coregistered onto the corresponding coordinates of the rat's CT scan and glycolytic activity was documented.

\section{Functional Testing}

The rats in both the tumor and control groups were evaluated for cognitive and functional deficits based on an automated rotarod test. ${ }^{16}$ The test involved progressive acceleration of a rotating spindle that scored the animal's ability to stay on the rod for a period of time. The initial starting speed of the rod was at $3 \mathrm{rpm}$ and increased in 0.5-rpm increments every 30 seconds. Animals were timed from the beginning of their movement on the rod to the time at which they fell off. Scores were assessed based on the time elapsed.

\section{Histological Studies}

Animals were killed after onset of hemiparesis or by 45 days after tumor implantation if no paresis had yet occurred. The procedures for the planned deaths were as follows: after administration of an intramuscular injection of $75 \mathrm{mg} / \mathrm{kg}$ ketamine hydrochloride and $15 \mathrm{mg} / \mathrm{kg}$ xylazine, perfusions were performed in which the thorax was opened, the right atrium was incised, and a cannula was inserted into the left ventricle. Normal saline followed by $10 \%$ paraformaldehyde was pumped through the rat's circulation, followed by an intraperitoneal injection of Euthanasia-6 solution (Veterinary Laboratories, Inc., Lenexa, $\mathrm{KS})$. Following death, the entire brain was dissected en bloc and placed in either 10\% paraformaldehyde or liquid nitrogen based on the histological methods to be used. After fixation, the brains were then sectioned and stained with $\mathrm{H} \& \mathrm{E}$. The presence of tumor and the pattern of infiltration were assessed, and intrinsic tumor characteristics and the effect of its presence on brainstem structure were recorded.

\section{Statistical Analysis}

Statistical analysis included comparisons of the onset of paraparesis between the tumor and control groups by using the log-rank test performed with JMP 5.1 software (SAS Institute, Inc., Cary, NC). The Kaplan-Meier curves of the three groups were graphed using Microsoft Excel 2003 software (Microsoft Corp., Redmond, WA). Probability values less than 0.05 were considered to be statistically significant.

\section{RESULTS}

The 9L and F98 tumor cells grew in 95\% of animals injected with the tumor cell suspension and resulted in statistically significant onsets of hemiparesis and durations of survival. The mean onset of hemiparesis in rats with 9L tumors was $17.1 \pm 0.64$ days and the median survival duration was $18.6 \pm 0.63$ days, whereas the rats with F98 tumors had a mean onset of hemiparesis of $15.9 \pm 0.48$ days and a median survival of $17.7 \pm 0.54$ days $(\mathrm{p}=0.001, \log$ rank test) after tumor implantation. Animals in the control group exhibited no neurological deficits by Day 60 (Fig. 2). Mild ipsilateral hind- and forelimb weakness rapidly progressed to severe paraparesis, usually within 24 hours (Table 1), and urinary and fecal incontinence along with visual otosis usually accompanied this event. Paralysis of motor 
ability was consistently observed in the hemisphere ipsilateral to the injection site.

Scoring of each animal's motor capabilities on the rotarod apparatus indicated that the ability of the rat to stay on the rotating spindle decreased with time. The mean performance for the control animals was 3 minutes \pm 40 seconds. Tumor-bearing rats began to show diminished performance by Day 5 (mean 2 minutes \pm 45 seconds) and regressed to less than 30 seconds by Day 15. The onset of paresis in these animals resulted in rotarod times of less than $30 \mathrm{sec}-$ onds, indicating tumor proliferation.

Among the imaging modalities available, high-resolution FDG-PET fused with CT scans best demonstrated spaceoccupying brainstem lesions, with acute tumor tissue demarcated by regions of high temperature and glycolytic activity (Fig. 3). Control rats did not show glycolytic activity in the brainstem (Fig. 4). Histopathological analysis confirmed the pontine tegmentum origin of the tumor and showed sheets of neoplastic cells with extensive invasion and compression of the brainstem (Fig. 5A) when compared with that of a control rat (Fig. 5B). Areas of marked ischemia and coagulative necrosis were seen within the tumor. Animals in the control group showed no functional, radiographic, or pathological signs of tumor.

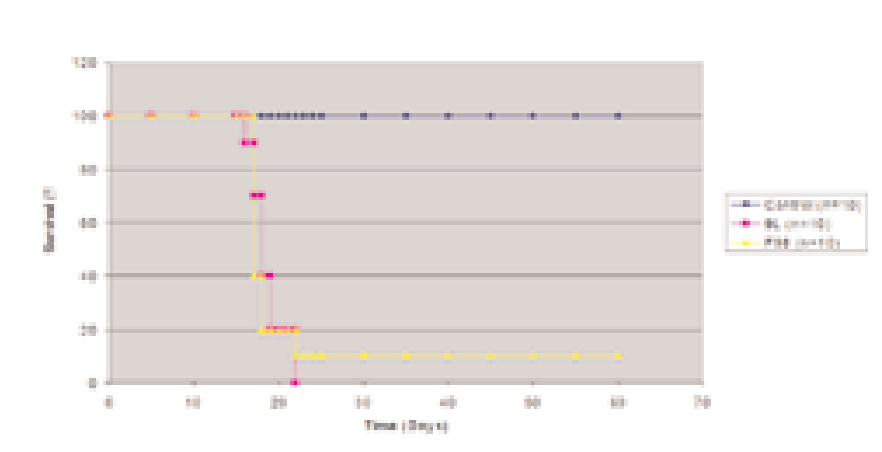

Fig. 2. Kaplan-Meier curve depicting survival rates for rats injected with DMEM (control), 9L gliosarcoma, or F98 glioma cells. $\mathrm{N}=$ number.

\section{DISCUSSION}

In this study we describe a novel animal model of brainstem tumors in rats in which cell suspensions are implanted by a single injection of 9L gliosarcoma and F98 glioma cells through a cannulated guide screw system. This tumor model will enable preclinical testing of novel treatment modalities, including local chemotherapy, radiation therapy, and antiangiogenic and immunomodulatory approaches, along with other combination therapies.

In formulating a brainstem tumor model, animal size was of particular interest because experimental treatment protocols in which brainstem drug delivery systems as well as imaging techniques are used need to be simulated for humans. Furthermore, the tumor model requires the ability to perform cell implantations in an easily and reliably reproducible fashion, with the resulting lesions displaying classic malignant features associated with diffuse brainstem tumors. Two of the few experimental tumor cell lines that are easily implantable in rats and that demonstrate similar features are the 9L gliosarcoma and F98 glioma cell lines. Both have been found to be syngeneic to Fischer 344 rats. These tumor lines are used extensively to investigate antitumor treatments in different organs, including the liver ${ }^{11}$ and kidney. ${ }^{10,15}$ Tumor induction occurred in all of these locations without an altered immune response and with a responder rate greater than $80 \%$. Our brainstem tumor model meets these expectations, with a $95 \%$ take rate and a tumor size of approximately $1.5 \mathrm{~cm}$ by 17 days postimplantation.

A review of the literature reveals no previously established brainstem tumor models that were investigated with real-time imaging. Recently, Jallo, et al., ${ }^{9}$ have reported an experimental tumor model that can be induced in neonatal rat pups by using 9L and F98 tumor cell suspensions injected into the brainstem. Tumors developed in 24 of 29 neonates, but the rate of maternal removal associated with the procedure varied between 5 to $20 \%$, with tumors failing to develop in five pups. Additionally, none of the pups underwent CT or PET imaging to deduce localized tumor growth in the brainstem.

Although researchers in this previous study demonstrat-

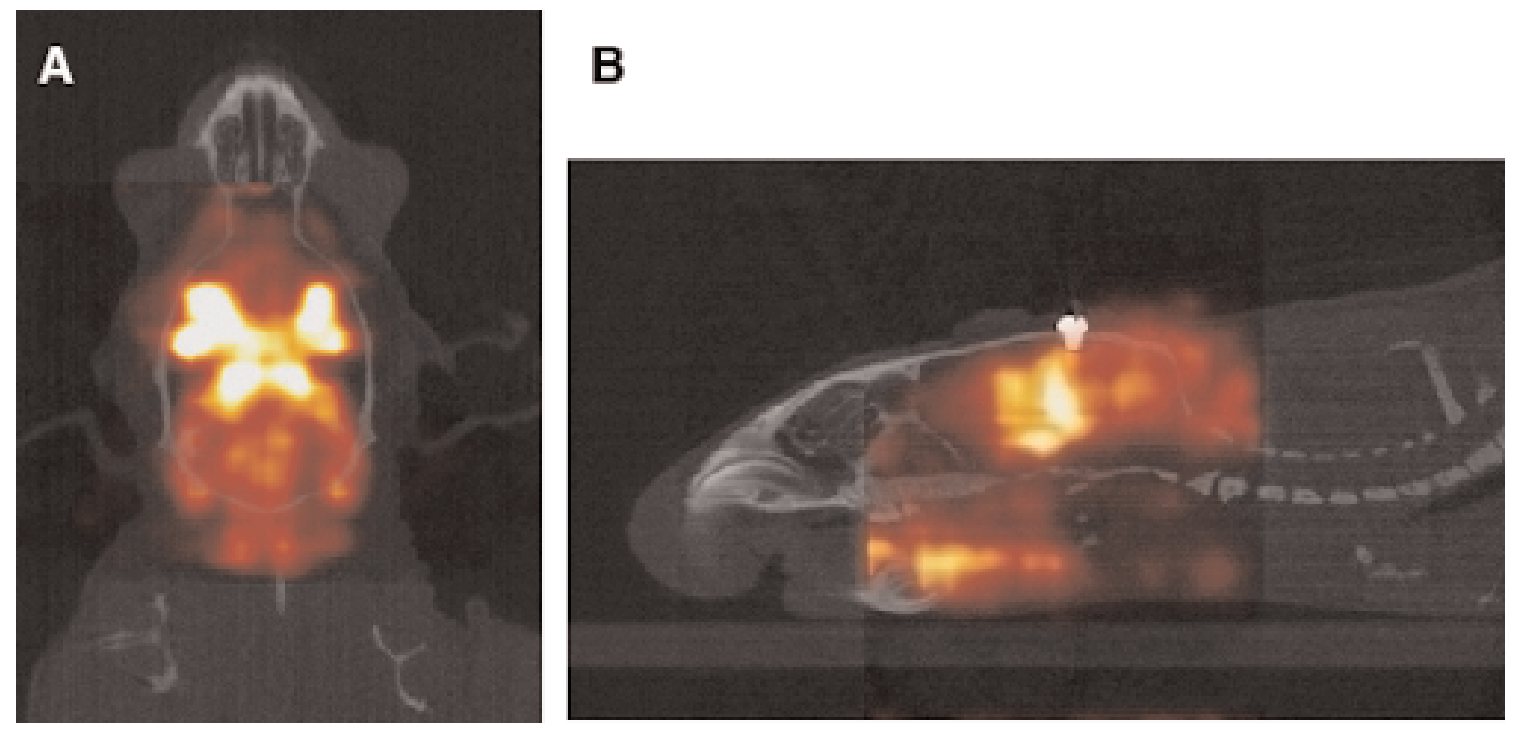

Fig. 3. Illustrative FDG-PET/CT axial (A) and sagittal (B) images with contrast demonstrating high glycolytic activity and brainstem tumor growth in a rat with an F98 tumor. 

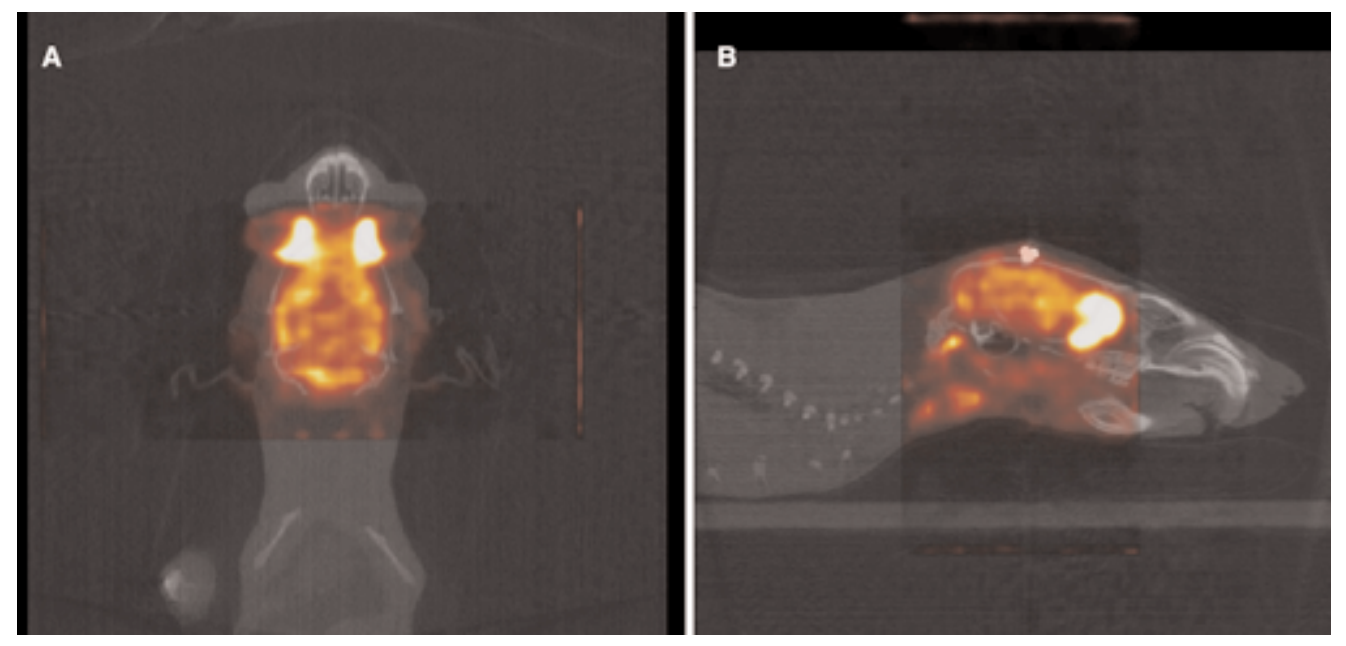

Fig. 4. Illustrative FDG-PET/CT axial (A) and sagittal (B) images with contrast demonstrating no glycolytic activity at the brainstem DMEM injection site in a control animal.

ed a technique for implanting brainstem tumors, the take rate of tumor growth and maternal removal of pups provided mixed results, and the advent of improved imaging modalities warrants a new model. Therefore, the rat brainstem tumor guide screw-based model with CT and FDGPET characterization provides an optimal framework for rapid and large-scale preclinical testing of new treatment paradigms. Because these advanced imaging modalities are the diagnostic studies of choice for human brainstem tumors, any animal model of these lesions must include such analysis. In our study, FDG-PET/CT coregistered imaging clearly demonstrated brainstem lesions with edema or infiltrative signal characteristics comparable to high-grade human brainstem tumors.

Weaknesses of this model include the use of nonhuman cell lines and the small sample size. Even though both tumor types appear homogeneous at early developmental stages, there are significant parametric image differences between tumors and normal brain tissue. Regardless of the cell line used, the lesions, in contrast to normal parenchyma, exhibit greatly elevated levels of thermal and glycolytic activity. This was especially apparent for the infiltrative F98 glioma cell line. Nevertheless, even in the absence of human cell lines, cultured rat glioma cell lines share histological and radiographic features with high-grade brainstem tumors and have been successfully used to model intracranial neoplasms. The sample size for this experiment was small; however, a group of 10 is acceptable given the 95\% take rate observed in the tumor-injected animals and the lack of deficits seen in the control rats. Despite its limitations, this model has important applications, including testing of novel treatment modalities such as stereotactic radiotherapy and local delivery of chemotherapy in a controlled experimental setting.

\section{CONCLUSIONS}

Although other CNS tumor models have been well stud-
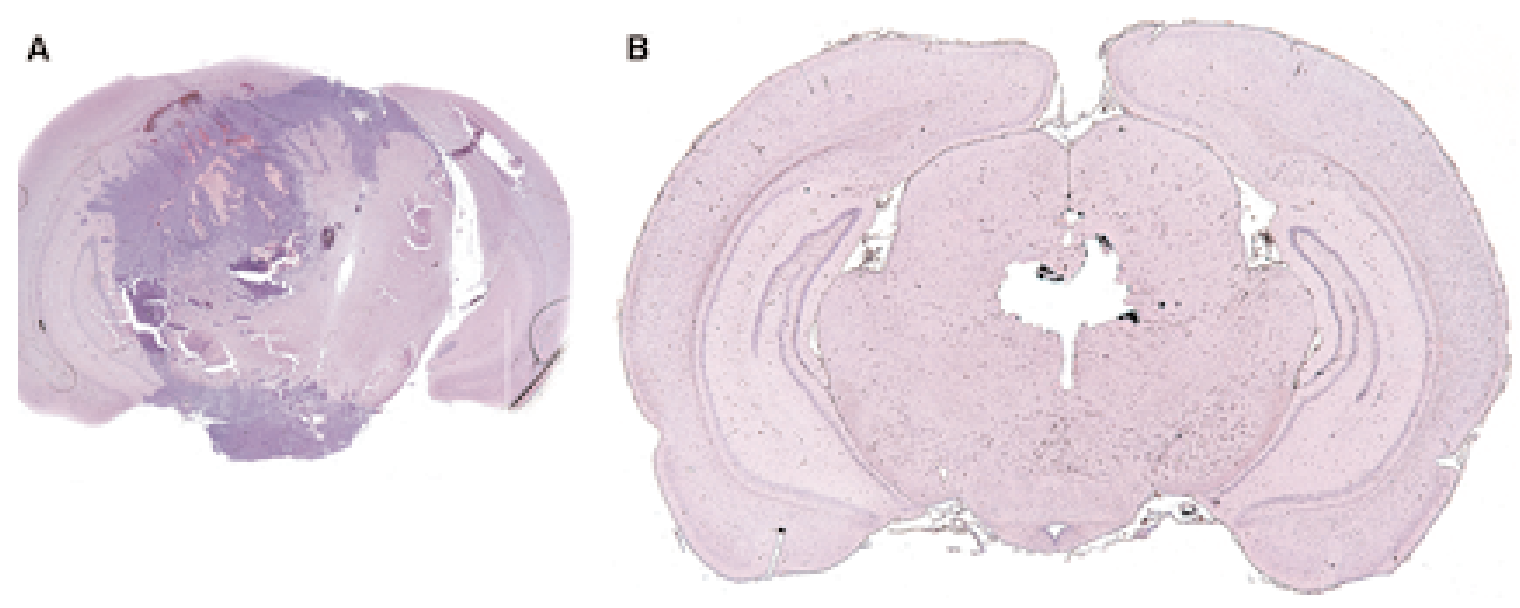

Fig. 5. A: Photomicrograph of a coronal cross-section of rat brain at the fourth ventricle level revealing a brainstem tumor, with marked ischemia and necrosis within the lesion and extensive invasion of the brainstem. B: Photomicrograph of a cross-section of the brainstem of a control rat. $\mathrm{H} \& \mathrm{E}$, original magnification $\times 1$. 
ied, ${ }^{18}$ this is the first reliable and reproducible animal brainstem tumor model complete with fused FDG-PET/CT imaging characterization. Clinical progression of cell proliferation was consistent and predictable, and the techniques and materials used are widely accessible. The histopathological and radiological characteristics of the brainstem 9L and F98 tumors are comparable to those of aggressive primary human brainstem tumors. The establishment of this tumor model represents an important step in the path toward developing new treatment paradigms that may prolong survival in patients with brainstem tumors.

\section{Acknowledgments}

We thank Dr. Martin Pomper and Mr. James Fox for radiological imaging, Mr. Young Cho for image reconstruction and coregistration, Mr. Norman Barker for histological studies, and Mr. Ian Suk for his illustrations.

\section{References}

1. Albright AL: Diffuse brainstem tumors: when is a biopsy necessary? Pediatr Neurosurg 24:252-255, 1996

2. Albright AL, Guthkelch AN, Packer RJ, et al: Prognostic factors in pediatric brain-stem glioma. J Neurosurg 6:751-755, 1986

3. Allen JC: Brain stem glioma. Neurol Neurosurg Update Series 4:2-7, 1983

4. Carson BS Sr, Wu Q, Tyler B, et al: New approach to tumor therapy for inoperable areas of the brain: chronic intraparenchymal drug delivery. J Neurooncol 60:151-158, 2002

5. Epstein F, Wisoff JH: Intrinsic brainstem tumors in childhood: surgical indications. J Neurooncol 6:309-317, 1988

6. Guarnieri M, Carson BS Sr: Chronic local therapy for brainstem tumors. Neurosurgery 54:1025-1027, 2004

7. Guerin C, Olivi A, Weingart JD, et al: Recent advances in brain tumor therapy: local intracerebral drug delivery by polymers. Invest New Drugs 22:27-37, 2004
8. Jallo GI, Biser-Rohrbaugh A, Freed, D: Brainstem gliomas. Childs Nervous System 20:143-153, 2004

9. Jallo GI, Penno M, Sukay L, et al: Experimental models of brainstem tumors: development of a neonatal rat model. Childs Nerv Syst 21:399-403, 2005

10. Jounaidi Y, Waxman DJ: Use of replication-condition adenovirus as a helper system to enhance delivery of P450 prodrug-activation genes for cancer therapy. Cancer Res 64:292-303, 2004

11. Lee FT Jr, Chosy SG, Naidu SG, et al: CT depiction of experimental liver tumors: contrast enhancement with hepatocyte-selective iodinated triglyceride versus conventional techniques. Radiology 203:465-470, 1997

12. Littman P, Jarrett P, Bilaniuk LT, et al: Pediatric brain stem gliomas. Cancer 45:2787-2792, 1980

13. Madajewicz S, Chowhan N, Tfayli A, et al: Therapy for patients with high grade astrocytoma using intraarterial chemotherapy and radiation therapy. Cancer 88:2350-2356, 2000

14. Occhiogrosso G, Edgar MA, Sandberg DI, et al: Prolonged convection-enhanced delivery into the rat brainstem. Neurosurgery 52:388-394, 2003

15. Rand RW, Snow HD, Elliott DG, et al: Thermo-magnetic surgery for experimental renal cancer. J Urol 128:618-620, 1982

16. Rozas G, Guerra MJ, Labandeira-Garcia JL: An automated rotarod method for quantitative drug-free evaluation of overall motor deficits in rat models of parkinsonism. Brain Res Brain Res Protoc 2:75-84, 1997

17. Storm PB, Moriarity JL, Tyler B, et al: Polymer delivery of camptothecin against 9L gliosarcoma: release, distribution, and efficacy. J Neurooncol 56:209-217, 2002

18. Weizsaecker M, Deen DF, Rosenblum ML, et al: The 9L rat brain tumor: description and application of an animal model. J Neurol 224:183-192, 1981

Manuscript received April 14, 2005.

Accepted in final form May 17, 2005.

Address reprint requests to: George I. Jallo, M.D., Department of Neurosurgery, Johns Hopkins Hospital, Harvey 811, 600 North Wolfe Street, Baltimore, Maryland 21287. email: gjallo1@jhmi.edu. 